\title{
Inductive Edge Sensors with Nanometer Accuracy for the European Extremely Large Telescope
}

\author{
D.Patel ${ }^{1}$, C.Néel $^{2}$ \\ ${ }^{1}$ Micro-Epsilon Messtechnik GmbH \& Co. KG. Koenigbacher Str. 15, 94496 Ortenburg. Germany, \\ ${ }^{2}$ FOGALE Nanotech, 125 rue de l'Hostellerie, 30900 Nimes, France. \\ diniel.patel@micro-epsilon.de \\ c.neel@fogale.com
}

\begin{abstract}
:
The primary mirror (M1) of the E-ELT consists of a mosaic of 798 hexagonal mirror segments, making this ELT on completion the largest segmented mirror telescope. Their relative positions will be measured by 4524 so-called edge sensors. Nanometric precision, low sensitivity to temperature and humidity fluctuations, high linearity and high reliability are the main properties of these three axes edge sensors. This paper presents the current status and progress of the development for these sensors in the framework of the contract signed between ESO (European Southern Observatory) and FAMES consortium - a collaboration between Micro-Epsilon (Germany) and Fogale Nanotech (France). It also talks about why the Edge Sensor uses an inductive measurement principle, manufactured with Embedded Coil Technology (ECT), been selected for the edge sensor rather than a capacitive measurement principle, namely the advantages of an inductive displacement sensor over capacitive displacement sensor for this special application. The paper also shows some of the challenges ahead for the successful completion of the task at hand, developing a sensor that satisfies the technical requirements from ESO as well as being able to produce the required quantity, in line with the signed contract.
\end{abstract}

Key words: Segmented mirror telescope, Inductive displacement sensor, ELT, Embedded Coil Technology (ECT), Edge Sensor 